\title{
Face Authenticated Hand Gesture Based Human Computer Interaction for Desktops
}

\author{
Venkat Mukthineni ${ }^{1}$, Rahul Mukthineni ${ }^{2}$, Onkar Sharma ${ }^{1}$, Swathi \\ Jamjala Narayanan ${ }^{1}$
}

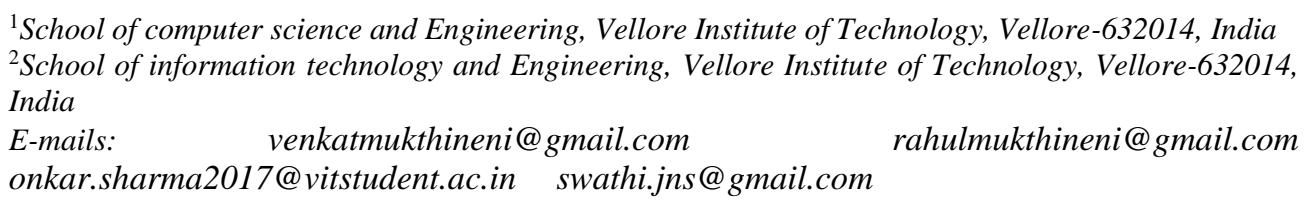

Abstract: Hand gesture detection and recognition is a cutting-edge technology that is getting progressively applicable in several applications, including the recent trends namely Virtual Reality and Augmented Reality. It is a key part of HumanComputer Interaction which gives an approach to two-way interaction between the computer and the user. Currently, this technology is limited to expensive and highly specialized equipment and gadgets such as Kinect and the Oculus Rift. In this paper, various technologies and methodologies of implementing a gesture detection and recognition system are discussed. The paper also includes the implementation of a face recognition module using the Viola-Jones Algorithm for authentication of the system followed by hand gesture recognition using CNN to perform basic operations on the laptop. Any type of user can use gesture control as an alternative and interesting way to control their laptop. Furthermore, this can be used as a prototype for future implementations in the field of virtual reality as well as augmented reality.

Keywords: Gesture recognition, gesture detection, human-computer interaction, Haar-cascade, convolution neural network, Viola-Jones Algorithm, ReLU activation, convolutional layer, max-pooling, $k$-Gaussian distribution.

\section{Introduction}

Face recognition is being used in many surveillance applications for security and privacy. Almost all the smart phones in the market have face recognition system to provide security for the data of the users. In a similar way, Face recognition can be extended to laptops. Face recognition is used to authenticate the valid user to use the application and when the user gets access to the laptop application, various tasks can be performed. It's an easy task for humans to detect the faces from the video but for computers it's quite challenging to detect faces from a noisy video and match the face in the video with the valid user image. Detection of human faces is difficult for the computer as users will be at different angles, distance from the camera of the desktop. 
The primary objective of face recognition is to differentiate different features in the input image and separate facial structures for the image. In this research ViolaJones Algorithm is used to detect the facial features from the input video and authenticate a valid user into our interaction system. Unrealistic background is eliminated from the video.

Hand gestures have been widely used by ancient people when they had have no language to communicate. Hand gestures at the present day could be characterized as an efficient natural communication methodology for people having problems in speaking and hearing. Hand gesture recognition systems have been applied in various fields and scenarios. Many hand gesture recognition systems have been developed, which help the users to play games and control the characters in the game. With the advancements in the human computer interaction, robots can be developed which can manage different applications at places where it's very hard to speak, e.g., robots can be used underwater where it's a very tough for humans to speak or write, and instead hand gestures can be used to control the system. In addition to this activity recognition systems [1] have been widely put into use.

In the past few years, a lot of research has been going on in the field of humancomputer interaction. Currently, two main methods have been developed for hand gesture detection and recognition. One method is by using wearable devices like hand gloves, electromagnetic gadgets, etc., and the other uses the Personal Computer (PC) version. However, the gadgets that are being used are quite expensive and not applicable for every condition, a lot of care needs to be taken for the device. Hand gesture based Human-Computer Interaction (HCI) can be categorized as follows:

\subsection{Wearable devices}

These are basically physical devices which consist of a lot of sensors present in them. Lots of input is taken by the sensors form the surrounding environment and actions are performed based on the input that is given to the sensors. Although the devices work well, they are quite expensive and difficult to use. Oculus Rift, Magic leap one, Kinet are a few examples of wearable devices.

\subsection{Non-wearable devices}

These strategies don't require the user to wear a different gadget on his/her hands yet rather catch a picture of the signal utilizing the webcam or a different camera and apply different image processing techniques. Few methods are given below [2]:

1. Background Removal methods: From the input image, the background is captured and is removed from the image. In this way, the part of the hand is captured with respect to the background and the part of hand alone is extracted. Based on the extracted feature gesture can be recognized.

2. Skin-color based detection: Skin colour of the user is taken into consideration, and the part of the hand is separated. But the problem with this is that some other features other than hand also get separated.

3. Skin temperature-based detection: Separate thermal cameras will be used to detect the thermal image of the hand. It works in many environments except at very high temperatures. 
4. Extraction of features: From the frames in the video or image, features are extracted, and hands are classified using classifiers.

5. Depth detection: Depth sensor is used to calculate the depth in the 2D image, thereby extracting the useful features from the image.

In this paper, we have implemented face recognition for user authentication and hand gestures to perform a few basic tasks on the laptop.

\section{Literature survey}

\subsection{Face recognition}

$\mathrm{V} \mathrm{i} \mathrm{k} \mathrm{r} \mathrm{a} \mathrm{m} \mathrm{and} \mathrm{Pa} \mathrm{d} \mathrm{m} \mathrm{a} \mathrm{va} \mathrm{th} \mathrm{i} \mathrm{[3]} \mathrm{propose} \mathrm{a} \mathrm{method} \mathrm{using} \mathrm{the} \mathrm{Viola-Jones}$ Algorithm to detect different facial features from the input image and extract them. Viola-Jones Cascade object detector is used to recognize features in the input face image. Prior to this method, many other methods have been proposed using different algorithms. $\mathrm{Z} \mathrm{h}$ a o, D e $1 \mathrm{l}$ e a $\mathrm{n} \mathrm{r}$ e a and $\mathrm{Ch}$ e $\mathrm{n}$ [4] propose a method using standard face recognizer, Kernel based tracking algorithm which uses Kalman filter. The KNN classifier of Earth Mover distance is used to classify the original face trajectories. But the accuracy of prediction is only 93\%. A face detection system to count people based on faces is developed in [5]. Rough edges are detected from the frame difference, and human faces are detected using chromatic features. Based on the colour coordinates from the input image, the skin colour region is detected and extracted face features are classified as fake or real. However, the accuracy of the proposed system is only $80 \%$. Illumination invariant and Principal Component Analysis (PCA) [6] can be used to overcome problems with light and pose of the person.

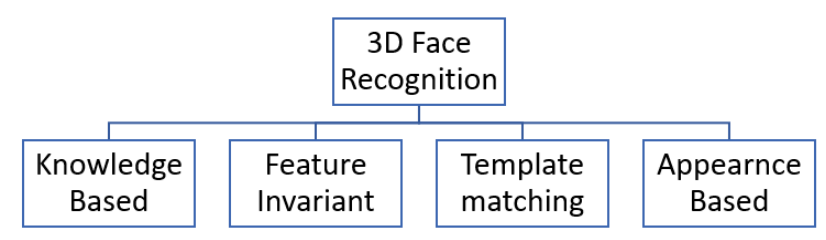

Fig. 1. Different exixting approaches for face recogition

Recognition of the human face under low light is one of the challenging tasks. Face recognition under low light can be developed by normalizing the image based on illumination, fusion of various features, matching based on distance transform, face representations based on local texture [7]. Gamma correction and difference of gaussian filtering, equalizing contrast are used as pre-processing steps. Multiorientation in the input image can be achieved by using Gabor filter [8]. Face and pose are detected from the input image. $\mathrm{P}$ a u 1 et al. [2] propose a method to recognize the face at a various distance, angles from camera with different background angles using Viola-Jones Algorithm. In order to recognize the faces from the input image, Principal Component Analysis (PCA) and Feed forward neural network can be used [9]. 


\subsubsection{Knowledge based methods}

This is one of the oldest methods for facial recognition. These methods mainly concentrate on the geometry of the human face. S c h e e $\mathrm{n} \mathrm{s} \mathrm{tra,} \mathrm{R} \mathrm{u} \mathrm{i} \mathrm{f} \mathrm{r} \mathrm{o} \mathrm{k} \mathrm{and}$ $\mathrm{V}$ e $1 \mathrm{t} \mathrm{k} \mathrm{a} \mathrm{m} \mathrm{p} \mathrm{[10]} \mathrm{explain} \mathrm{all} \mathrm{the} \mathrm{existing} \mathrm{methods} \mathrm{of} 2 \mathrm{D}$ facial recognition methods and the improvements that can be made for effective recognition. K o t r o p o u 1 o s and $\mathrm{P}$ i t a s [11] propose a rule-based facial detection approach on ACTS M2VTS dataset.

\subsubsection{Feature invariant methods}

A different approach to knowledge-based methods is using facial features like nose, ears, mouth, etc. Textures from the human face are extracted using second-order statistics method [12]. Facial features are computed on 16×16 sub-images. $\mathrm{S}$ irohey $\mathrm{S}$ a ad [13] use clutter to segment facial features from the images and elliptical structures are used to separate the head portion from an image with a noisy background. Based on the edge map information, an ellipse is fitted across the head part so that it gets differentiated from the background. A b b a s, A h m e d and A b $\mathrm{b}$ a s [14] have developed a face recognition model based on the coherent parts of the face and has achieved a recognition rate of $96.4 \%$ on FRGGCv2 dataset.

\subsubsection{Template matching methods}

These methods are used for detection of localization of faces using the correlations between image stored and the input image from the user [15]. A standard pattern of face is loaded priorly using a parameterized function. Facial features like nose, ears, mouth, etc, are calculated based on the correlation values. But the problem with this method arrives with orientation, pose and angle of the face. With the variation in the illumination in the image, features are identified. In the method proposed by $\mathrm{J}$ i n et al. [16] skin pixels in the RGB image are detected by luminance-conditional distribution model, later skin region is extracted in the form of rectangles by performing morphological operations.

\subsubsection{Appearance based methods}

Unlike the above approaches, appearance-based methods require a lot of data to train. A two-class pattern classifier is used to classify the facial and non-facial images [17]. Colour cue-based classification is far better than geometry-based methods as it can overcome hue, saturation and resolution problems in the image. M u t e $1 \mathrm{o}$ et al. [18] used a Haar-like feature algorithm to detect faces which overcomes the problem of occlusion. Facial features extraction using two-dimensional reduced principal component analysis which removes redundancies in the 2D image matrix rows [18]. Later rows, and columns are compressed and features are extracted from the image and classified. J a m e e 1 [19] considers facial features as a set of blocks and applies Direct Cosine Transforms (DCT) on those blocks. PCA is used in the place of inverse DCT to obtain high accuracy on ORL dataset. Blocks of facial images are modified by HMM. $\mathrm{H}$ a s h e $\mathrm{m} \mathrm{i} \mathrm{a}$ and $\mathrm{G} \mathrm{h}$ a r a h b a g h [20] have developed a face recognition system by decomposing images using a $2 \mathrm{D}$ wavelet transformation with 
eigenvalues and a radial basis function is used as a classifier. $\mathrm{G}$ a o and $\mathrm{L}$ e e [21] extend the SIFT algorithm with probabilistic similarity at different viewpoints to recognize a face. Affine SIFT differentiates 2D image and 3D face. However, this method doesn't work well at different poses. Log likelihood between the gallery and probe images are obtained using Sum of Squared Distribution (SSD). S o m p u r a and $\mathrm{G} \mathrm{u} \mathrm{p} \mathrm{t} \mathrm{a} \mathrm{[22]} \mathrm{propose} \mathrm{a} \mathrm{method} \mathrm{using} \mathrm{PCA} \mathrm{and} \mathrm{Low} \mathrm{Binary} \mathrm{Pattern} \mathrm{(LBP)} \mathrm{to}$ extract the global and local images from the images. Finally, classification is done using Back Propagation Multilayer Perceptron (BPMLP) with 93\% accuracy. A $1 \mathrm{~s}$ $\mathrm{h}$ e b a $\mathrm{i}, \mathrm{Pr}$ e mara $\mathrm{nte}$ and $\mathrm{V}$ i a 1 [23] propose a hybrid method in which facial features are segmented manually. Local representations are calculated using Gabor transforms of areas that are extracted. F e $\mathrm{n} \mathrm{g} \mathrm{x} \mathrm{i} \mathrm{a} \mathrm{n} \mathrm{g} \mathrm{[24]} \mathrm{use} \mathrm{wavelet}$ transform and local binary pattern that is regionally weighted directionally for face recognition. To improve the LBP operator, regional directed weighted LBP is exploited, and horizontal component prior principle is used.

\subsection{Hand gesture recognition}

Gesture recognition of hand is of two categories, i.e., static gesture and dynamic gesture. Static gesture classification uses the hand image of a person, whereas dynamic gesture classification takes many factors like velocity, the orientation of hand, shape, etc., into consideration.

$\mathrm{H}$ a s a $\mathrm{n}$ and $\mathrm{M}$ i s h r a [25] propose a method using Gaussian multivariate distribution function. $\mathrm{Ng}$ and $\mathrm{R}$ a $\mathrm{ng}$ a $\mathrm{n}$ a $\mathrm{th}$ [26] propose a method which extracts blobs from hand using Fourier descriptors and then the sequence of the image is acquired. HMM and RNN are used for obtaining the performance of gesture recognition. From the experiments performed, HMM and RNN combination outperformed the yield of continuous HMM. N o $1 \mathrm{k} \mathrm{e} \mathrm{r}$ and $\mathrm{R}$ i t t e r [27] have developed a gesture recognition system which uses the position of finger tips from the input image. A two-stage approach is developed in which finger location from the input video image is located using an Artificial Neural Network (ANN) followed by estimation of 3D configuration of the hand. $\mathrm{K}$ a o and $\mathrm{F} \mathrm{a} \mathrm{h} \mathrm{n} \mathrm{[28]} \mathrm{use} \mathrm{hand} \mathrm{motion}$ trajectories and HMM classifier with $96 \%$ accuracy. ANN training is comparatively slow. D h u l e and $\mathrm{N}$ a g r a r e [29] have developed a real-time motion control of mouse using the change in pixel values of RGB colours in the input video. $\mathrm{L}$ i n and D i n g [30] use histograms of oriented gradients features and information of motion trajectory in a natural environment. After detection of hand by HOG, hand is localized in the video stream. Later, the extraction of motion trajectory information from consecutive hand gestures is done. Recognition is done based on the Mahalanobis distance between the input image and database of standard gestures.

The main step in hand gesture recognition is segmentation [31]. Many segmentation techniques have been proposed till date. Based on the difference between the skin colour and external environment, the segmentation of hands is done. The problem with skin colour-based segmentation is that every object in the colour of skin like face also gets segmented. Discontinuity of grey scale value in the image can also be used to segment the hand portion. This is done using edge detection [32]. Nearest neighbour classifier is used to classify the input image into six classes. 
$\mathrm{H} \mathrm{u}$ a $\mathrm{g}, \mathrm{H} \mathrm{u}$ and $\mathrm{Ch}$ an $\mathrm{g}$ [33] have developed a combination of SVM and Gabor filter-based recognition system. Hand gesture features are acquired by convolving Gabor filters. Dimensionality of feature space is reduced using Principal Component Analysis (PCA). Shape based features [34] can be used for gesture classification. For robust recognition of gesture at different backgrounds, illumination compensation technique is used. To avoid false detections, skin colour segmentation is used. To capture the image, a metal oxide semiconductor image sensor camera is used. After pre-processing, $\mathrm{YCbCr}$ colour space is used to perform skin colour segmentation. I $\mathrm{s} 1 \mathrm{a} \mathrm{m}$ et al. [35] propose an advanced method for hand gesture recognition using CNN's. various data argumentation techniques like rescaling, rotating, height and width modification, re-sizing are applied on the data with an accuracy of 97.12\%. $\mathrm{R}$ a $\mathrm{m}$ et al. [36] have developed a method to control PowerPoint presentation using hand gestures. Based on the number of active fingers, hand gestures are recognized and slides can be changed. However, it's accuracy is better only at illuminated environments. RGB-D sensor is used by $\mathrm{P}$ a 1 a c i o s et al. [37] which works well on complex backgrounds and occlusions. Colour and semantic information is used to identify the count of hands in the image. Based on the spread of the fingers in the image, 10 different hand gestures are recognized. Segmentation is done in four phases: hand segmentation, feature extraction, static and dynamic gesture classification. T r i g u e i r o s, R i b e i r o and R e i s [38] use a SVM trained model to recognize static gestures and HMM trained model for dynamic gesture recognition. Depth information is used for posture recognition by $\mathrm{P}$ o u 1 a r a k i s and $\mathrm{K}$ a t s a v o u n id is, S t e rg o i s and K a t s a voun id i s i [39]. Apexshaped structure is located in hand contour. The advantage of this method is that it overcomes the challenge of partially merged fingers. Fourier Descriptors of contours is used for recognition. $\mathrm{X} \mathrm{u}$ et al. [40] propose a system for complex backgrounds which uses a single colour camera. For pre-processing, colour image is converted into hue image. Skin image is segmented from original image.

To reduce the redundancy in the information of the EMG signal and perform hand gesture recognition, principal component analysis and GRNN network is used in [41], which has achieved a 95.1\% recognition rate. $\mathrm{S}$ u et al. [42] use depth vision to label the classes of EMG data for hand gesture recognition. It has the capability to recognize the hand gesture without prior data labelling. A $\mathrm{me} \mathrm{u} \mathrm{r,} \mathrm{K} \mathrm{h} \mathrm{a} 1 \mathrm{If} \mathrm{a}$ and B o u h 1 e 1 [43] use Long Short Term Memory (LSTM) network on sequential time series data from Leap motion and propose a complex and efficient hand gesture recognition model using Hybrid Bidirectional LSTM and have achieved 90\% recognition rate on LeapGestureDB and RIT datasets. Z h o u, L I and W a n g [44] propose a 3D hand gesture recognition system, which is robust to motions with temporal variations. It has the capability to identify 8 different gestures with $93.9 \%$ accuracy with a 700Kbyte model. T a o et al. [45] fuse hand silhouettes, positions of figures and hand motions for hand gesture recognition. Local and multimodal features are optimized by multi-view learning-based fusion of features. S a t h a k u $\mathrm{m}$ a $\mathrm{r}$ et al. [46] propose an angular velocity method which performs hand gesture detection on real-time 3D sensor data and is capable of recognizing static and dynamic hand gestures. T r a n et al.[47] uses 3DCNN to detect fingertips and perform hand gesture 
spotting and recognition. Hand gesture recognition system is proposed by $\mathrm{A} h \mathrm{l}$ a w a $\mathrm{t}$ et al. [48] use contours around the palm and Hue Saturation Values (HSV) of the hand are used.

\section{Proposed method}

\subsection{Face recognition}

A facial recognition module is implemented for the authentication of users to the application. In this approach, Viola-Jones Algorithm is used as the training rate of the algorithm is very high. Scales are located by invariant detectors and feature selection process is effective [49]. Viola Jones works well in high contrast differences between background and the face and also can detect faces at an angle of 60 degrees approximately.

For validating the results, we have created our own dataset, which includes 101 images or pictures of the users. Pictures are taken from various angles and included in the dataset identifying the user and giving access to the system. The final goal of the trained system is to crop the face of the user and check whether the face of the user in the video captured by camera is matching with the trained images from the dataset or not.

In the training process, patterns in the image are identified. Haar-cascade classifier is used to identify the faces in the image and extract the unique feature from it. Each pixel of the input image is taken and is classified whether the selected pixel is part of the face or not.

\subsubsection{Rectangular features}

Instead of classification based on each and every pixel, Viola-Jones Algorithm uses rectangular features to classify the parts. Bi-rectangular features show the difference between the pixel summations of the two rectangular parts. Tri-rectangular features are the difference between the central rectangular summation and the external rectangular summation. In the last rectangle (iii) shown in the Fig. 2, difference in pixel summation of diagonal rectangles is represented.

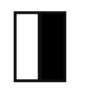

(i)

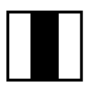

(ii)

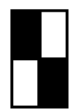

(iii)

Fig. 2. Haar features

The main classifier used for face detection is Haar cascade classifier. Facial features are recognized using these Haar features. The output of each feature is calculated by dividing the summation of black pixels with respect to white pixel summation. The detection process starts from the top left end of the input image from the video, and it ends at the right bottom end corner of the image. Rectangular Haar features are used repeatedly to detect the faces. 


\subsubsection{Internal image}

Computation of rectangular features is done using integral image. Each rectangle contains four edges and the pixel sum is calculated based on the edge values.

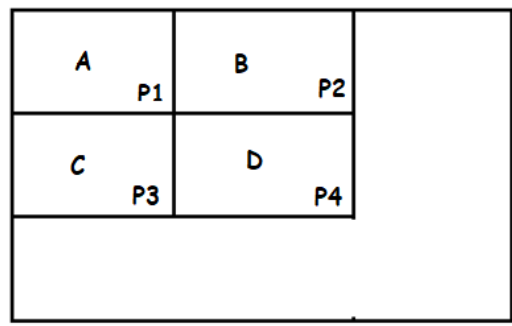

Fig. 3. Integral image calculation

$$
\begin{gathered}
\mathrm{P} 1=A, \\
\mathrm{P} 2=A+B, \\
\mathrm{P} 3=A+C, \\
\mathrm{P} 4=A+B+C+D,
\end{gathered}
$$

where $\mathrm{P} 1, \mathrm{P} 2, \mathrm{P} 3, \mathrm{P} 4$ are the pixel intensities at different rectangular regions $A, B, C$, $D$ which is calculated as shown above. The value of $D$ can be calculated by obtaining the difference between the sum of diagonal pixels. It is depicted in the next equation:

$$
\mathrm{P} 1+\mathrm{P} 4-\mathrm{P} 2-\mathrm{P} 3=D \text {. }
$$

\subsubsection{AdaBoost}

Classifier training and selection of features is done using variant AdaBoost [50]. It is a combination of weak classifiers which finally forms a strong classifier. The weights of the classifier are changed with iterations so that the final obtained classifier is strong. Strong classifier equation is represented as

$$
G(x)=\alpha G_{1}(x)+\alpha G_{2}(x)+\alpha G_{3}(x)+\ldots
$$

\subsubsection{Training}

For training purpose, 100 images are collected. This training dataset consists of correct or target attributes so that when a valid user enters the frame, the users face will get recognized and will have access to the system. For pattern analysis of face structure, Local Binary Pattern Histogram (LBPH) is used. Image is converted to greyscale image, later a neighbourhood of centre pixel is selected. For this pixel, LBP value is calculated and stored in the form of an array. Finally, Linear SVM is used for classification.

Finally, when training of the dataset is completed, the model is tested with a valid user. 


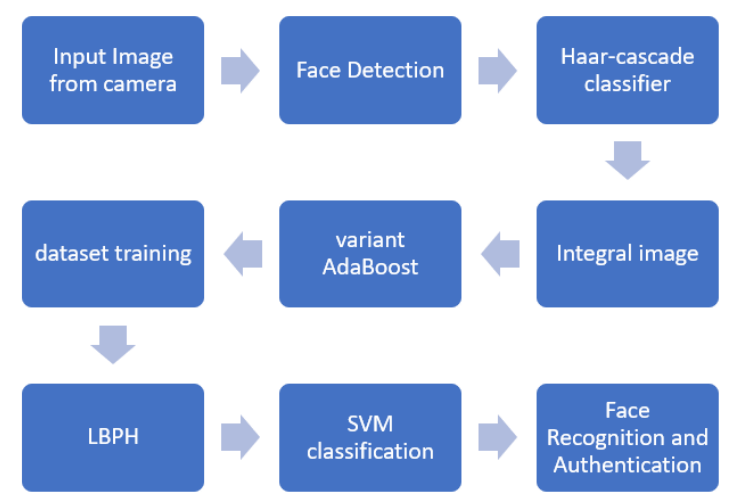

Fig. 4. Workflow of face recognition

\subsection{Hand gesture recognition}

The three main sub processes in hand gesture recognition is segmentation, tracking and recognition of gesture.

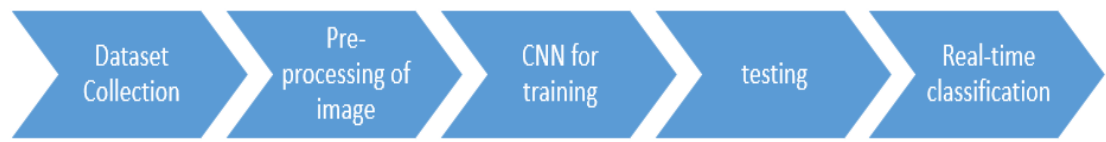

Fig. 5. Workflow of hand gesture recognition

\subsubsection{Training data}

For the training of the neural network, 1000 images of 10 different gestures are used. This data is used to recognize the gestures from the input video of the laptop's camera. For efficient classification of hand gestures, training data with a less complex background is utilized. The alignment of hand in the data is vertical.

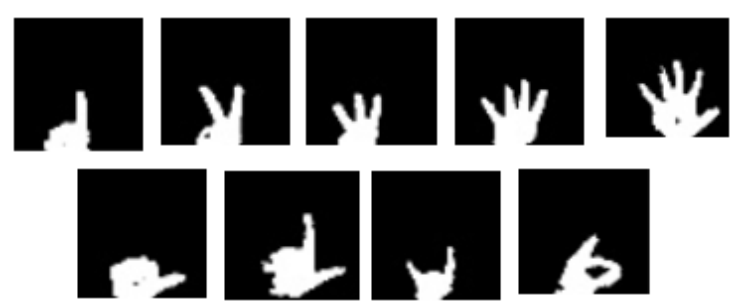

Fig. 6. Various hand gestures from training set

\subsubsection{Pre-processing of the data}

To increase the efficiency of hand gesture recognition, pre-processing of images needs to be done. Background subtraction method [51] is used to eliminate the background in the image. k-Gaussian distribution is used to remove the background and extract only the part of the hand from the image. Background noise is removed by using morphological transformations. 


\subsubsection{Dataset}

10,000 images are used as training data and 500 images as test data which is taken from hand gesture recognition database by Leap Motion, Kaggle.

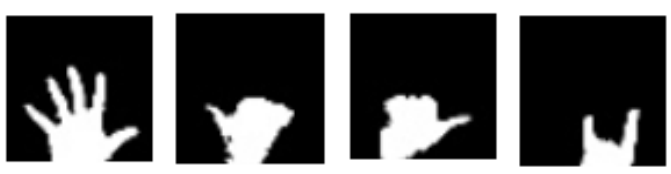

Fig. 7. Few samples from the test data

\subsubsection{CNN}

Batch size of 32 is used; 10 different classes of input data are created; $20 \%$ of data is used for validation. All the training and validation images are loaded into the OpenCV memory during the training process. A new TensorFlow session is created and all the parameters are declared. Weights and biases are declared and trained. For the training process, seven convolutional layers, seven max-pooling layers, and two fully connected layers are used. Initially the input image is sent into the first convolutional layer, the output of this convolutional layer is sent as input to the maxpool layer where max-pooling is done using a matrix of dimension $2 \times 2$. ReLU activation is performed on the output of the max-pool layer. This process is repeated seven times and the resultant output is a layer with $10 \times 10$ feature maps. This output is sent as input to the fully connected layer of size $1024 \times 1024$. The parameters of the convolutional layers are shown in the Table 1.

Table 1. Convolutional layer parameters

\begin{tabular}{|c|c|c|}
\hline Layer & Filter size & Number of filters \\
\hline Convolution 1 & 2 & 32 \\
\hline Convolution 2 & 2 & 32 \\
\hline Convolution 3 & 2 & 64 \\
\hline Convolution 4 & 2 & 32 \\
\hline Convolution 5 & 2 & 64 \\
\hline Convolution 6 & 2 & 32 \\
\hline Convolution 7 & 2 & 64 \\
\hline
\end{tabular}

OpenCV is used to access the camera object. Then resizing of the frame to $700 \times 400$ is performed. For each gesture, the image is blurred and converted into HSV colour space. A binary image is created in which all the skin pixels will be white, and rest pixels are converted into black. Skin colour area is increased by using dilation and erosion.

For the real-time prediction of hand gestures, image is resized, and the preprocessing is done on the input video image. Pre-processing is done similar to the training data pre-processing, However, the input to the network is of different shape, so reshaping is done. Image is flipped and rotated so that it matches with the training data [37]. Image is compared until 50 frames. 


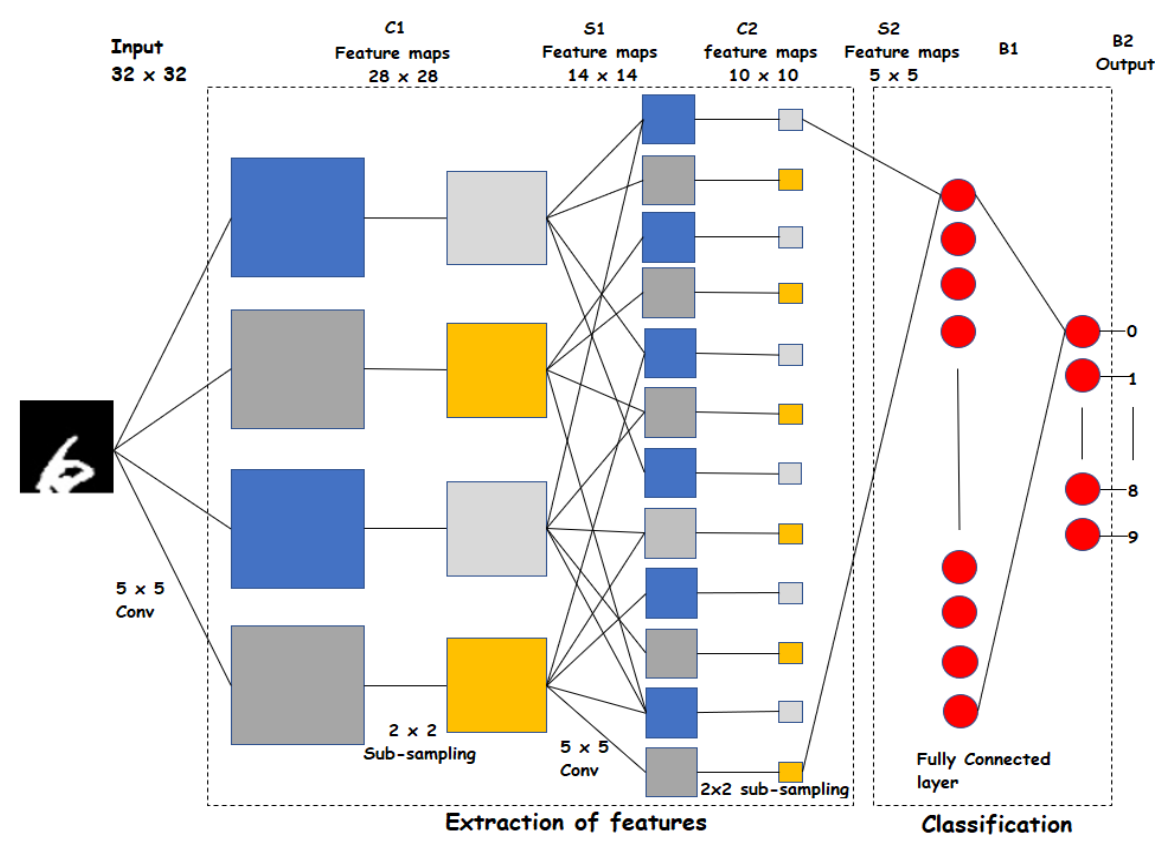

Fig. 8. Architecture of training CNN

\subsection{Implementation}

Python IDE Spyder is used for implementation. The Python libraries such as cv2, Os, Glob, Scikit, PIL, Time, Tensorflow, MATH, Random, time for image preprocessing, mathematical calculations etc., are used in this process. Firstly, the authentication of the user is done using facial recognition. If a valid user is recognized, the user is given access to the system. Once the authentication part is completed, hand gestures are recognized. Based on these hand gestures predefined apps will be opened.

Any app installed on the computer can be opened by giving the complete path of the executable file to the system. We have used the system for opening notepad, Ubuntu and Chrome. This can be extended to any other app.

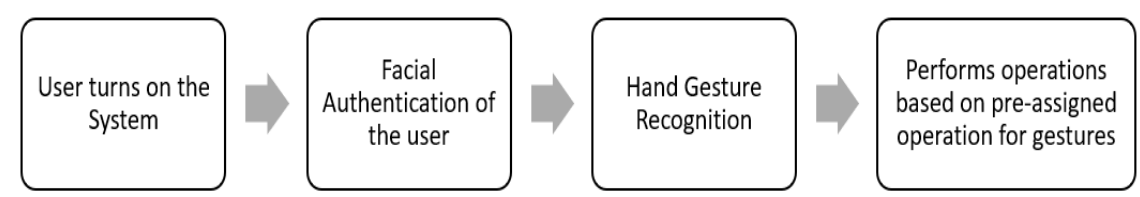

Fig. 9. Working of the developed system

\section{Results}

The whole working process and the output is shown in Fig. 10. Fig. 10a is the snapshot of the face recognition module in action. The webcam opens and detects the face. The face is then matched with the one that it has been trained with, and if it continuously matches for 50 frames then, it is recognized and the next module opens. 
Fig. 10b is the snapshot of the gesture detection module. The webcam opens and a green rectangle is shown where the gesture should be. Fig. 10c is the snapshot of the frame after the background has been subtracted. Fig. 10d contains snapshots of various gestures that can be recognized by the system after background subtraction, and thresholding. The frame in the left bottom corner represents the image after background subtraction and the one beside it is the image after thresholding. Fig. 10e shows notepad opening on gesture number 3. Similarly, other applications can be associated with the rest of the gestures.

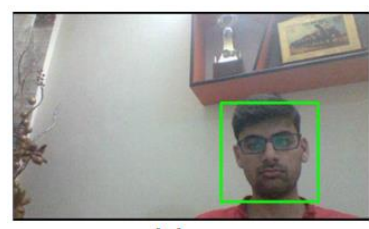

(a)

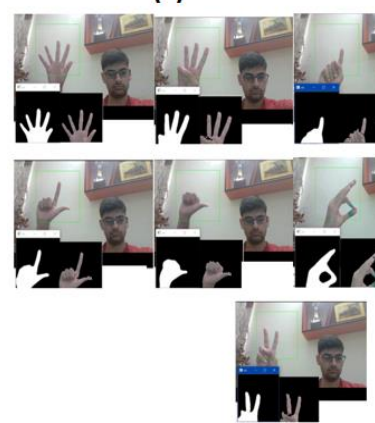

(d)

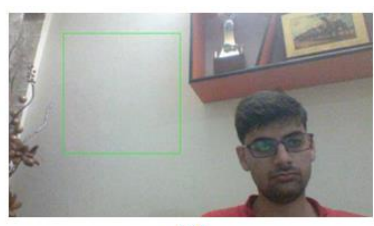

(b)

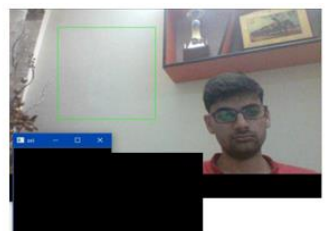

(c)

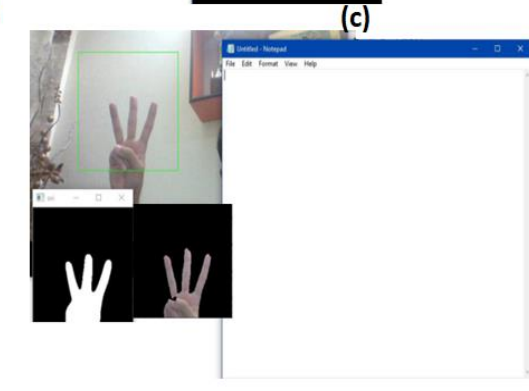

(e)

Fig. 10. Results

Fig. 11 shows the accuracy and loss of hand gesture recognition on our own dataset. We trained our hand gesture recognition model on a Hand gesture recognition database acquired by Leap motion which is publicly available on Kaggle. The model is trained for 30 epochs. The accuracy and the loss of training data is shown in Fig. 12.
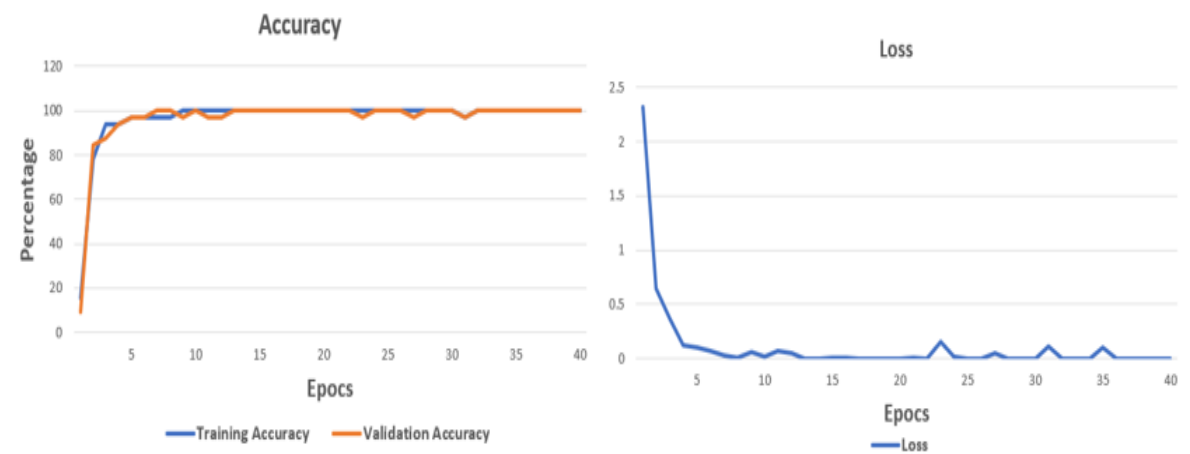

Fig. 11. Accuracy and loss on our own dataset 


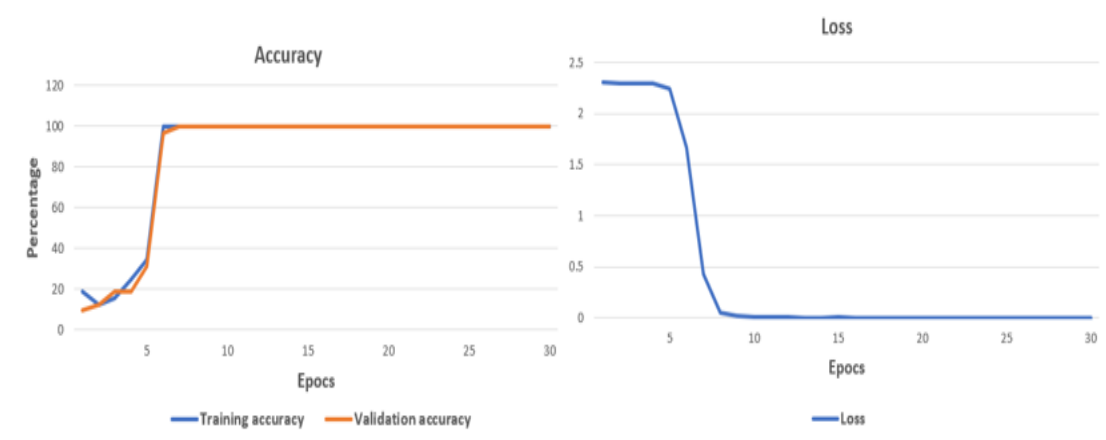

Fig. 12. Accuracy and loss on hand gesture recognition databases by Leap motion, Kaggle

\section{Conclusion}

A complete gesture recognition system with facial authentication has been discussed. The system is capable of recognizing a total of nine gestures. Face detection and recognition is performed by using the Viola-Jones Algorithm. Convolution neural network is used as the base of the system which is trained using a dataset of 10,000 unique images. The current system is proposed as a prototype and has a lot of potential to be used in various fields in the future.

\section{Future work and its applications}

Our work can be improvised in future so that this interaction system will support all the features in the laptop. Future enhancements in this interaction system helps physically disabled persons who can't move from their place to control the laptop and perform all the operations that a typical advanced user can perform. AR and VR are new emerging technologies in the market which are on great demand. HCI plays a vital role in this field. The proposed model can be implemented in AR and VR to add new features into the system and also to enhance the usability of the AR and VR system. Hand gesture recognition can also be used in Robotics to train robots to work like humans. This system can also be used in military purposes as a secret code to communicate with each other. The efficiency of the Viola-Jones Algorithm can be increased by concentrating on noisy backgrounds. This helps to classify the human faces accurately.

\section{References}

1. K e e, Y. J., M. N. S. Z a i n u d i n, M. I. Id r i s, R. H. R a m l e e, M. R. K a m a r u d i n. Activity Recognition on Subject Independent Using Machine Learning. - Cybernetics and Information Technologies, Vol. 20, 2020, No 3, pp. 64-74.

2. T a $n$ m o y, P., U. A. $S h$ a $m \mathrm{~m}$ i, M. U. A h m e d, R. R a h $\mathrm{m}$ a n, S. K o b a s h i, A. R. A h a d. A Study on Face Detection Using Viola Jones Algorithm in Various Backgrounds, Angles and Distances. - International Journal of Biomedical Soft Computing and Human Sciences: The Official Journal of the Biomedical Fuzzy Systems Association, Vol. 23, 2018, No 1, pp. 27-36. 
3. V i k r a m, K., S. P a d m a v a t h i. Facial Parts Detection Using Viola Jones Algorithm. - In: Proc. of 4th International Conference on Advanced Computing and Communication Systems (ICACCS'17), IEEE, 2017, pp. 1-4.

4. Z h a o, X., E. D e 11 e a n d r e a, L. C h e n. A People Counting System Based on Face Detection and Tracking in a Video. - In: Proc. of 6th IEEE International Conference on Advanced Video and Signal Based Surveillance, IEEE, 2009, pp. 67-72.

5. Ch e n, T., Y. C h a o, H. C h e n, D. J. W a n g, Y. L. K u o. A People Counting System Based on Face-Detection. - In: Proc. of 4th International Conference on Genetic and Evolutionary Computing, IEEE, 2010, pp. 699-702.

6. P a t e 1, Y., A. P a n d e y, M. P a r e k h, S. N a y a k. Automatic Facial Recognition and Surveillance System. - International Journal for Research in Applied Science and Engineering Technology, 2018, pp. 2321-9653.

7. A ru $1 \mathrm{ku}$ m a r, C. V., P. V i v e k a n a n d a n. Multi-Feature Based Automatic Face Identification on Kernel Eigen Spaces (KES) under Unstable Lighting Conditions. - In: Proc. of 2015 International Conference on Advanced Computing and Communication Systems, IEEE, 2015, pp. $1-5$.

8. K a r t h i k a, R., L. P a r a m e s w a r a n. Study of Gabor Wavelet for Face Recognition Invariant to Pose and Orientation. - In: Proc. of International Conference on Soft Computing Systems, Springer, New Delhi, 2016, pp. 501-509.

9. D e s h p a n d e, N. T., S. R a v i s h a $\mathrm{n}$ k a r. Face Detection and Recognition Using Viola-Jones Algorithm and Fusion of PCA and ANN. - Advances in Computational Sciences and Technology, Vol. 10, 2017, No 5, pp. 1173-1189.

10. S c h e e n s t r a, A., A. R u i f r o k, R. C. V e $1 \mathrm{t} \mathrm{k} \mathrm{a} \mathrm{m} \mathrm{p.} \mathrm{A} \mathrm{Survey} \mathrm{of} \mathrm{3rd} \mathrm{Face} \mathrm{Recognition}$ Methods. - In: Proc. of International Conference on Audio- and Video-Based Biometric Person Authentication, Springer, Berlin, Heidelberg, 2005, pp. 891-899.

11. K o t r o p o u 1 o s, C., I. P i t a s. Rule-Based Face Detection in Frontal Views. - In: Proc. of IEEE International Conference on Acoustics, Speech, and Signal Processing, IEEE, Vol. 4, 1997, pp. 2537-2540.

12. A u g u s t e i j n, M. F., T. L. S k u f c a. Identification of Human Faces through Texture-Based Feature Recognition and Neural Network Technology. - In: Proc. of IEEE International Conference on Neural Networks, IEEE, 1993, pp. 392-398.

13. S i r o h e y, S. A. Human Face Segmentation and Identification. Semantic Scholar, 1998.

14. A b b a s, H. H., B. Z. A h m e d, A. K. A b b a s. 3D Face Factorisation for Face Recognition Using Pattern Recognition Algorithms. - Cybernetics and Information Technologies, Vol. 19, 2019, No 2, pp. 28-37.

15. R i z v i, Q. M., B. G. A g a r w a l, R. B e g. A Review on Face Detection Methods. - Journal of Management Development and Information Technology, 2011.

16. J i n, Z., Z. L o u, J. Y a n g, Q. S u n. Face Detection Using Template Matching and Skin-Color Information. - Neurocomputing, Vol. 70, 2007, No 4-6, pp. 794-800.

17. N i s h i n a, Y., M. A. A h a d, J. K. T a n, H. S. K i m, S. I s h i k a w a. A Robust Face Tracking Method by Employing Color-Based Particle Filter. - International Journal of Biomedical Soft Computing and Human Sciences: The Official Journal of the Biomedical Fuzzy Systems Association, Vol. 16, 2011, No 1, pp. 127-134.

18. M u t e 1 o, R. M., L. C. K h o r, W. L. W o o, S. S. D 1 a y. Two-Dimensional Reduction PCA: A Novel Approach for Feature Extraction, Representation, and Recognition. - In: Visualization and Data Analysis. Vol. 6060. 2006.

19. J a m e e 1, S. Face Recognition System Using PCA and DCT in HMM. - Int. J. Adv. Res. Comput. Commun. Eng., Vol. 4, 2015, No 1, pp. 13-8.

20. H a s h e m i, V. H, A. A. G h a r a h b a g h. A Novel Hybrid Method for Face Recognition Based on 2nd Wavelet and Singular Value Decomposition. - American Journal of Networks and Communications, Vol. 4, 2015, No 4, pp. 90-94.

21. G a o, Y., H. J. L e e. Viewpoint Unconstrained Face Recognition Based on Affine Local Descriptors and Probabilistic Similarity. - Journal of Information Processing Systems, 2015.

22. S o m p u r a, M., V. G u p t a. An Efficient Face Recognition with ANN Using Hybrid Feature Extraction Methods. - International Journal of Computer Applications, Vol. 11, 2015, No 4.

23. A $1 \mathrm{~S}$ h e b a n i, Q., P. Pre mar a n t e, P. J. Vi a 1., 2014, 166. 
24. F e $\mathrm{n}$ g x i a n g, W. Face Recognition Based on Wavelet Transform and Regional Directional Weighted Local Binary Pattern. - Journal of Multimedia, Vol. 9, 2014, No 8.

25. H a s a n, M. M., P. K. M i s h r a. Features Fitting Using Multivariate Gaussian Distribution for Hand Gesture Recognition. - International Journal of Computer Science \& Emerging Technologies IJCSET, Vol. 3, 2012, No 2, pp. 73-80.

26. N g, C. W., S. R a n g a n a t h. Real-Time Gesture Recognition System and Application. - Image and Vision Computing, Vol. 20, 2002, No 13-14, pp. 993-1007.

27. N o 1 k e r, C., H. R i t t e r. Visual Recognition of Continuous Hand Postures. - IEEE Transactions on Neural Networks, Vol. 13, 2002, No 4, pp. 983-994.

28. K a o, C. Y., C. S. F a h n. A Human-Machine Interaction Technique: Hand Gesture Recognition Based on Hidden Markov Models with Trajectory of Hand Motion. - Procedia Engineering, 2011, pp. 3739-3743.

29. D h u 1 e, C., T. N a g r a r e. Computer Vision Based Human-Computer Interaction Using Color Detection Techniques. - In: Proc. of 4th International Conference on Communication Systems and Network Technologies, IEEE, 2014, pp. 934-938.

30. L i n, J., Y. D i n g. A Temporal Hand Gesture Recognition System Based on Hog and Motion Trajectory. - Optik, Vol. 124, 2013, No 24, pp. 6795-6798.

31. S u n, J. H., T. T. J i, S. B. Z h a n g, J. K. Y a n g, G. R. J i. Research on the Hand Gesture Recognition Based on Deep Learning. - In: Proc. of 12th International Symposium on Antennas, Propagation and EM Theory (ISAPE), IEEE, 2018, pp. 1-4.

32. L i o n n i e, R., I. K. T i m o t i u s, I. S e t y a w a n. An Analysis of Edge Detection as a Feature Extractor in a Hand Gesture Recognition System Based on Nearest Neighbour. - In: Proc. of International Conference on Electrical Engineering and Informatics, IEEE, 2011, pp. 1-4.

33. H u a n g, D. Y., W. C. H u, S. H. C h a n g. Vision-Based Hand Gesture Recognition Using PCA+ Gabor Filters and SVM. - In: Proc. of International Conference on Intelligent Information Hiding and Multimedia Signal Processing, IEEE, 2009, pp. 1-4.

34. G u p t a, A., V. K. S e h r a w a t, M. K h o s 1 a. FPGA Based Real Time Human Hand Gesture Real Recognition System. - Procedia Technology, 2012, pp. 98-107.

35. I s 1 a m, M. Z., M. S. H o s s a i n, R. U 1 i s 1 a m, K. A n d e r s s o n. Static Hand Gesture Recognition Using Convolutional Neural Network with Data Augmentation. - In: Proc. of International Conference on Informatics, Electronics \& Vision (ICIEV) and International Conference on Imaging, Vision \& Pattern Recognition (icIVPR), 2019, pp. 324-329.

36. R a m R a j e s h, J., R. S u d h a r s h a n, D. N a g a r j u n a n, R. A a r t h i. Remotely Controlled PowerPoint Presentation Navigation Using Hand Gestures. - In: Proc. of International Conference on Advances in Computer, Electronics and Electrical Engineering, 2012.

37. P a 1 a c I o s, J. M., C. S a g ü é s, E. M o n t i j a n o, S. L 1 or e n t e. Human-Computer Interaction Based on Hand Gestures Using RGB-D Sensors. - Sensors, Vol. 13, 2013, No 9, pp. 11842-11860.

38. T r i g u e i r o s, P., F. R i b e i r o, L. P. R e i s. Generic System for Human-Computer Gesture Interaction. - In: Proc. of IEEE International Conference on Autonomous Robot Systems and Competitions (ICARSC'14), IEEE, 2014, pp. 175-180.

39. P o u 1 a r a k i s, S., I. K a t s a v o u n i d i s i. Finger Detection and Hand Posture Recognition Based on Depth Information. - In: Proc. of IEEE International Conference on Acoustics, Speech and Signal Processing (ICASSP'14), 2014, pp. 4329-4333.

40. X u, Y., J. G u, Z. T a o, D. W u. Bare Hand Gesture Recognition with a Single Color Camera. - In: Proc. of International Congress on Image and Signal Processing, IEEE, 2009, pp. 1-4.

41. Q i, J., G. J i a n g, G. L i, Y. S u n, B. T a o. Surface EMG Hand Gesture Recognition System Based on PCA and GRNN. - Neural Computing and Applications, Vol. 32, 2020, No 10, pp. 6343-6351.

42. S u, H., S. E. O v u r, X. Z h o u, W. Q i, G. F e r r i g n o, E. De M o m i. Depth Vision Guided Hand Gesture Recognition Using Electromyographic Signals. - Advanced Robotics, 2020, pp. 1-13.

43. A m e u r, S., A .B. K h a 1 I f a, M .S. B o u h 1 e 1 . A Novel Hybrid Bidirectional Unidirectional LSTM Network for Dynamic Hand Gesture Recognition with Leap Motion. - Entertainment Computing, 2020, 35, p. 100373. 
44. Z h o u, F., X. L i, Z. W a n g. Efficient High Cross-User Recognition Rate Ultrasonic Hand Gesture Recognition System - IEEE Sensors Journal, 2020.

45. S o n g, T., H. Z h a o, Z. L i u, H. L i u, Y. H u, D. S u n. Intelligent Human Hand Gesture Recognition by Local-Global Fusing Quality-Aware Features. - Future Generation Computer Systems, 2020.

46. $\mathrm{S} h$ a $\mathrm{th}$ a k u m a r, V. A., C. P e n g, J. H a n s be rge r, L. C a o, S. M e a c h a m, V. B la ke1 y. Design and Evaluation of a Hand Gesture Recognition Approach for Real-Time Interactions. - Multimedia Tools and Applications, 2020, pp. 1-24.

47. T r a n, D.-S., N.-H. H o, H.-J. Y a n g, E.-T. B a e k, S.-H. K i m, G. L e e. Real-Time Hand Gesture Spotting and Recognition Using RGB-D Camera and 3D Convolutional Neural Network. Applied Sciences, Vol. 10, 2020, No 2, 722.

48. A h 1 a w a t, S., V. B a t r a, S. B a n e r j e e, J. S a h a, A. K. G a r g. Hand Gesture Recognition Using Convolutional Neural Network. - In: Proc. of International Conference on Innovative Computing and Communications, Springer, Singapore, 2019, pp. 179-186.

49. V i j a y a l a k s m i, K. A. Comparison of Viola-Jones and Kanade-Lucas-Tomasi Face Detection Algorithms. - Oriental Journal of Computer Science and Technology, Vol. 10, 2017, No 10.

50. F r e u n d, Y., R. E. S c h a p i r e. A Desicion-Theoretic Generalization of On-Line Learning and an Application to Boosting. - In: Proc. of European Conference on Computational Learning Theory, Springer, Berlin, Heidelberg, 1995, pp. 23-37.

51. Z i v k o v i c, Z., F. V a n D e r H e i j d e n. Efficient Adaptive Density Estimation per Image Pixel for the Task of Background Subtraction. - Pattern Recognition Letters, Vol. 27, 2006, No 7, pp. 773-780.

Received: 23.04.2020; Second Version: 30.09.2020; Accepted: 16.10.2020 